\title{
Metformin Reduces Cardiometabolic Risk Factors in People at High Risk for Development of Type 2 Diabetes and Cardiovascular Disease
}

\section{Petya Kamenova ${ }^{1 *}$, Iliyana Atanasova ${ }^{2}$ and Georgi Kirilov ${ }^{3}$}

${ }^{1}$ Department of Diabetology, Clinical Center of Endocrinology and Gerontology, Medical University, Sofia, Bulgaria

${ }^{2}$ Clinical Laboratory, Clinical Center of Endocrinology and Gerontology, Medical University, Sofia, Bulgaria

${ }^{3}$ Laboratory of radioimmune assay, Clinical Center of Endocrinology and Gerontology, Medical University, Sofia, Bulgaria

\begin{abstract}
Background: The aim of the study was to investigate the effect of metformin on cardiometabolic risk factors as visceral obesity, hyperinsulinaemia, insulin resistance, dyslipidaemia and arterial hypertension in hyperinsulinaemic normal glucose tolerant people with metabolic syndrome who represent a high-risk group for development of type 2 diabetes and cardiovascular disease.
\end{abstract}

Methods: 52 participants (20 men/32 women) of mean age $40.1 \pm 14.2$ yrs were included in an open-label prospective one year observational clinical study in which cardiometabolic risk markers at three months intervals were followed. Metformin was applied at a mean dose of $2.55 \pm 0.2 \mathrm{~g}$ daily on a usual diet and physical activity.

Results: Body mass index, waist circumference, fasting serum insulin and homeostasis model assessment of insulin resistance significantly reduced at 6,9 month and at 1 year of metformin treatment $($ all $p<0.001)$. Fasting plasma glucose and 2-h post glucose load serum insulin significantly decreased at 3, 6, 9 month and at 1 year (both $p<0.001)$. Triglycerides, systolic and distolic blood pressure significantly decreased at 9 month and at 1 year (all $p<0.001)$. LDL cholesterol significantly reduced at 9 month and at 1 year $(p=0.001)$. HDL cholesterol significantly increased at 1 year of metformin treatment $(p<0.001)$.

Conclusion: Metformin reduces cardiometabolic risk factors in hyperinsulinaemic, normal glucose tolerant people with metabolic syndrome and could be considered as a therapeutic option for prevention of type 2 diabetes and cardiovascular disease.

Keywords: Metformin; Type 2 diabetes; Obesity; Metabolic syndrome; Cardiometabolic risk factors; Normal glucose tolerance; Hyperinsulinaemia

\section{Introduction}

Metabolic Syndrome (MS) is defined as a constellation of cardiometabolic risk factors that are associated with an increased risk of Cardiovascular Disease (CVD) and type 2 diabetes (T2D) [1]. T2D and CVD affect increasing number of young and social active people, so that the prevention of these diseases is a strategical aim of health systems all over the world, the main part of which is treatment of the MS. There is no a definitive therapy of the MS unless reduction of the cardiometabolic risk factors as visceral obesity, dyslipidaemia, arterial hypertension and hyperglycaemia [2]. Even in individuals with genetic predisposition for development of T2D, the environmental factors like diet and physical activity could influence the development of diabetes. Consequently, the change in lifestyle aimed to a reduction of body weight is the first and most important step for diabetic prevention [24]. In individuals who do not get the desirable result, the addition of medical treatment is a helpfull alternative $[5,6]$.

Hyperinsulinaemia is considered as the earliest stage of development of T2D and a core defect unifying the cardiovascular cluster of the MS. Hyperinsulinaemia is a metabolic antecedent or cause rather than consequence of obesity and dietary and pharmacologic approach that reduce high insulin levels could promote weight loss [5].

Metformin is established like a first step therapy of T2D, because it targets insulin resistance and hyperinsulinaemia and fulfills the treatment goals-a good glycaemic control and a reduction of cardiometabolic risk. It is the only antidiabetic medication that has improved prognosis like a primary end point in the randomized controlled trial (UKPDS) [7]. Metformin reduces the incidence of T2D and CVD risk factors in individuals with impaired fasting blood glucose and impaired glucose tolerance $[3,4,8-10]$.

At present, metformin is one of the main therapeutic options in the polycystic ovary syndrome, which landmarks are insulin resistance and hyperinsulinaemia defining higher incidence of MS and increased cardiovascular risk [11-15]. Having in mind, the protective cardiometabolic effects of metformin beyond glycaemic control, the research and clinical interest is pointed at its implication in individuals with normal glucose tolerance at high risk for development of T2D and CVD. The data about the effect of metformin on CVD risk factors in those people are inconclusive and demanding more investigations $[5,6,10,16-23]$.

The aim of the present study was to investigate the effect of metformin on cardiometabolic risk factors as visceral obesity, hyperinsulinaemia, insulin resistance, dyslipidaemia and arterial hypertension in people who have hyperinsulinaemia, normal glucose

*Corresponding author: Petya Kamenova, Department of Diabetology, Clinical Center of Endocrinology and Gerontology, Medical University, No 2, Zdrave str. 1431, Sofia, Bulgaria, Tel: +359-888343952; E-mail: kamenovap@abv.bg

Received October 22, 2014; Accepted November 16, 2014; Published November 18,2014

Citation: Kamenova P, Atanasova I, Kirilov G (2014) Metformin Reduces Cardiometabolic Risk Factors in People at High Risk for Development of Type 2 Diabetes and Cardiovascular Disease. J Diabetes Metab 5: 470 doi:10.4172/21556156.1000470

Copyright: (c) 2014 Kamenova P, et al. This is an open-access article distributed under the terms of the Creative Commons Attribution License, which permits unrestricted use, distribution, and reproduction in any medium, provided the original author and source are credited. 
tolerance and MS, representing a high-risk group for development of T2D and CVD.

\section{Materials and Methods}

\section{Study participants}

Fifty two participants (20 men, 32 women) attending Department of Diabetology of Clinical Center of Endocrinology and Gerontology were included in the study. The inclusion criteria were: 1) age-between 18-59 years; 2) MS according to the International Diabetes Federation definition with a necessary requirement central obesity defined as waist circumference $\geq 80 \mathrm{~cm}$ for women and $\geq 94 \mathrm{~cm}$ for men and two of the following: triglycerides $\geq 1.7 \mathrm{mmol} / \mathrm{l}$ and/or High Density Lipoprotein (HDL) cholesterol $<1.03$ for men and $<1.29 \mathrm{mmol} / \mathrm{l}$ for women and/or specific treatment, systolic blood pressure $\geq 130 \mathrm{mmHg}$ and/or diastolic blood pressure $\geq 85 \mathrm{mmHg}$ and/or specific treatment, fasting plasma glucose $\geq 5.6 \mathrm{mmol} / \mathrm{l}[24]$; 3) normal glucose tolerance during $75 \mathrm{~g}$ oral glucose tolerance test (OGTT) defined as fasting plasma glucose $<6.1 \mathrm{mmol} / \mathrm{l}$ and 2-h Post Glucose Load (PGL) plasma glucose $<7.8$ $\mathrm{mmol} / \mathrm{l}$; 4) hyperinsulinaemia during OGTT-fasting serum insulin $>25$ $\mathrm{mIU} / \mathrm{l}$ and/or 2-h PGL serum insulin $>3$ times from baseline; 5) failure to loose weight with conventional medical care - caloric restriction plus participation in regular exercise at a minimum level of $150 \mathrm{~min}$ moderate-intensity sessions per week in prior year; 6) no presence of CVD or diseases of the endocrine system that are post related with MS; 7) no family history for diabetes; 8) no contraindications for metformin treatment. Characteristics of persons with hyperinsulinaemia, normal glucose tolerance and MS is presented in Table 1.

\section{Study procedure}

At baseline visit case history, physical examination, questionnaire about quality of life including diet and physical activity and blood measurements of 90 people were performed and 52 of them fulfilling the inclusion criteria were involved in an open-label prospective one year observational clinical study. Participants were admitted to the Department of Diabetology of Clinical Center of Endocrinology and Gerontology at baseline visit and at 3, 6,9 month and at 1 year visits to perform all anthropometric, clinical and blood measurements and to check diet, physical activity and drug administration. Side effects of metformin treatment were recorded at 3,6,9 month and at 1 year.

\begin{tabular}{|l|c|}
\hline Variables & Mean \pm SD \\
\hline Sex $($ men / women) & $20 / 32$ \\
\hline Body weight $(\mathrm{kg})$ & $97.5 \pm 18.5$ \\
\hline Body Mass Index $\left(\mathrm{kg} / \mathrm{m}^{2}\right)$ & $32.3 \pm 5.2$ \\
\hline Waist circumference $(\mathrm{cm})$ & $102.8 \pm 14.3$ \\
\hline Systolic blood pressure $(\mathrm{mm} \mathrm{Hg})$ & $131 \pm 18$ \\
\hline Diastolic blood pressure $(\mathrm{mmHg})$ & $85 \pm 11$ \\
\hline Fasting plasma glucose $(\mathrm{mmol} / \mathrm{l})$ & $5.40 \pm 0.53$ \\
\hline 2-h PGL plasma glucose $(\mathrm{mmol} / \mathrm{l})$ & $5.74 \pm 1.27$ \\
\hline Fasting serum insulin $(\mathrm{mlU} / \mathrm{l})$ & $24.1 \pm 15.3$ \\
\hline 2-h PGL serum insulin $(\mathrm{mlU} / \mathrm{l})$ & $62.6 \pm 43.2$ \\
\hline HOMA-IR & $5.74 \pm 3.77$ \\
\hline Total cholesterol $(\mathrm{mmol} / \mathrm{l})$ & $5.78 \pm 0.76$ \\
\hline LDL cholesterol $(\mathrm{mmol} / \mathrm{l})$ & $3.61 \pm 0.76$ \\
\hline HDL cholesterol $(\mathrm{mmol} / \mathrm{l})$ & $1.10 \pm 0.34$ \\
\hline Triglycerides $(\mathrm{mmol} / \mathrm{l})$ & $2.60 \pm 1.74$ \\
\hline
\end{tabular}

Table 1: Characteristics of persons with hyperinsulinaemia, normal glucose tolerance and metabolic syndrome; $\mathrm{PGL}=$ post glucose load; HOMA-IR= homeostasis model assessment of insulin resistance; LDL= low density lipoprotein $\mathrm{HDL}=$ high density lipoprotein
The participants had on disposal a constant telephone contact with investigators for any questions and problems occurred during the study. Body weight, Body Mass Index (BMI), waist circumference, systolic blood pressure, diastolic blood pressure, OGTT, homeostasis model assessment of insulin resistance (HOMA-IR), serum lipidstotal cholesterol, HDL cholesterol, Low Density Lipoprotein (LDL) cholesterol and triglycerides at baseline and at 3, 6, 9 month and at 1 year were followed.

Written informed consent was obtained from all participants. The study protocol was approved by the local Ethical Committee of the Clinical Center of Endocrinology and Gerontology.

\section{Drug administration}

It was an open-label study in which metformin, tablets of $1000 \mathrm{mg}$, was applied at an initial dose of $500 \mathrm{mg}$ in the middle of the dinner for 5 days, titrated in 5 days intervals by $500 \mathrm{mg}$ at lunch, at breakfast and at dinner consecutively to the maximal of $3 \mathrm{~g}$ or until side effects occurred, when the previous well tolerated dose was kept constant during the follow-up. The mean dose of metformin was $2.55 \pm 0.2 \mathrm{~g}$, taken at breakfast, at lunch and at dinner in the middle of the meal. Questions about diet referred to the type of food, food ingredients, timing of the meal and daily calory intake. Questions about physical activity concerned the duration and regularity of physical activity at home, at work and during sport. Participants were advised to keep on their usual diet and physical activity throughout the whole study. They were not given individual dietary and physical activity recommendations. Individuals who were taking lipid lowering and antihypertensive drugs continued their use to the end of the observation.

\section{Clinical methods}

The body weight was measured to the nearest $0.1 \mathrm{~kg}$ with the participants in light clothing and without shoes by using a digital weighing scale (Seca, Germany). Height was measured to the nearest $0.1 \mathrm{~cm}$ using a commercial stadiometer (Momert Co, Ltd, Hungary) with the participants barefoot. BMI was calculated as body weight $(\mathrm{kg})$ divided by squared height $\left(\mathrm{m}^{2}\right)$. Waist circumference was measured using a non-elastic tape to the nearest $0.1 \mathrm{~cm}$ in the middle between lowest rib and the iliac crest in inspiration and expiration position and the mean value was taken. Systolic and diastolic blood pressure was measured with the use of manual sphygmomanometer on the left arm in a sitting position after at least 10 min of rest. Mean value was determined from two independent measurements with a 3 min interval between readings.

\section{Analytical methods}

Blood samples for glucose, insulin and lipids were taken after a 12-hour overnight fast. They were immediately centrifuged and analysed. OGTT with measurement of plasma glucose and serum insulin on fasting and at 1,2 and 3-h after a $75 \mathrm{~g}$ glucose load was performed. Plasma glucose was defined by a glucose oxidase method on biochemical analyzer (Cobas Integra, Roche Diagnostics, Switzerland). Serum insulin was estimated by an immunoradiometric method (Insulin IRMA kit, Immunotech, Beckman Coulter, Czech Republic, reference range 2-25 mIU/l) with analytical sensitivity $0.49 \mathrm{mIU} / \mathrm{l}$ and functional sensitivity $1.35 \mathrm{mIU} / \mathrm{l}$. The assay exhibit extremely low cross reactivities with human proinsulin, C-peptide, Des-31, 32 proinsulin, Lispro (Humalog ${ }^{\circledast}$ ) and 55\% cross-reactivity with Des-64,65 proinsulin. The intra-assay coefficient of variation was found below $4.0 \%$, the inter-assay coefficient of variation was found below $4.8 \%$. HOMAIR was calculated as fasting plasma glucose $(\mathrm{mmol} / \mathrm{l}) \mathrm{x}$ fasting serum 
Citation: Kamenova P, Atanasova I, Kirilov G (2014) Metformin Reduces Cardiometabolic Risk Factors in People at High Risk for Development of Type 2 Diabetes and Cardiovascular Disease. J Diabetes Metab 5: 470 doi:10.4172/2155-6156.1000470

Page 3 of 6

insulin (mIU/l) / 22.5 [25]. Serum lipids-total cholesterol, triglycerides and HDL cholesterol were measured by an enzymatic method on biochemical analyzer (Cobas Integra, Roche Diagnostics, Switzerland). LDL cholesterol was calculated using Friedewald equation.

\section{Statistical methods}

Statistical analysis was done using statistical package for social science (SPSS, version 14 for Windows, Chicago, Il, USA). Data are presented as mean \pm SD and as percentages. To evaluate differences between baseline and follow-up variables one-way analysis of variance (ANOVA) with repeated measures or Friedman test according to a normal or a nonparametric distribution of the tested variable were used. Correlation between two variables was assessed by Pearson or Spearman correlation analysis as appropriate. Shapiro-Wilk test was used for normality. A significant level of $\mathrm{p}<0.05$ was considered.

\section{Results}

The significant effect of metformin on body weight, BMI (Figure 1) and waist circumference (Figure 2) was noticed at 6,9 month and at 1 year of metformin treatment. There was no a statistically significant difference between body weight, BMI and waist circumference at 9 month and at 1 year compared to the 6 month. Body weight was reduced by $7.9 \%$ at 6 month, by $10.8 \%$ at 9 month and by $12.4 \%$ at 1 year of metformin treatment. The mean body weight reduction was 7.7 $\mathrm{kg}$ (from 2 to $20 \mathrm{~kg}$ ) at 6 month, $10.5 \mathrm{~kg}$ (from 4 to $20 \mathrm{~kg}$ ) at 9 month and $12.1 \mathrm{~kg}$ (from 4 to $22 \mathrm{~kg}$ ) at 1 year. The correlation between body

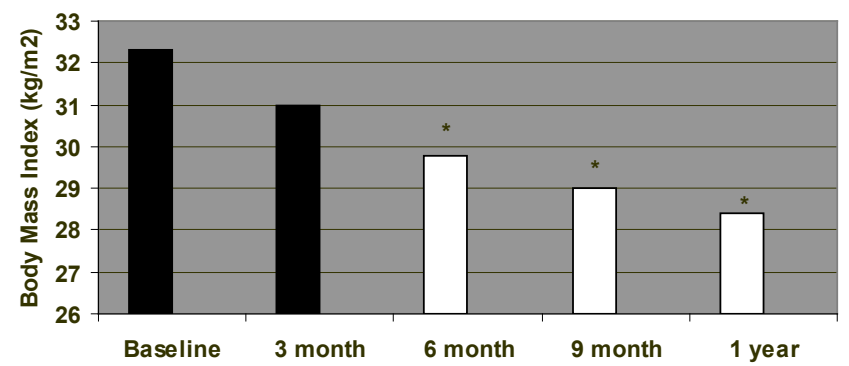

*significant difference versus baseline.

Figure 1: Effect of metformin on body mass index. BMI $32.3 \pm 5.2 \mathrm{~kg} / \mathrm{m}^{2}$ significantly reduced at 6 month $29.9 \pm 4.8 \mathrm{~kg} / \mathrm{m}^{2}(\mathrm{p}=0.012)$, at 9 month 29.0 $\pm 4.8 \mathrm{~kg} / \mathrm{m}^{2}(p=0.001)$ and at 1 year $28.4 \pm 5.0 \mathrm{~kg} / \mathrm{m}^{2}(p<0.001) ; B M l=$ body mass index.

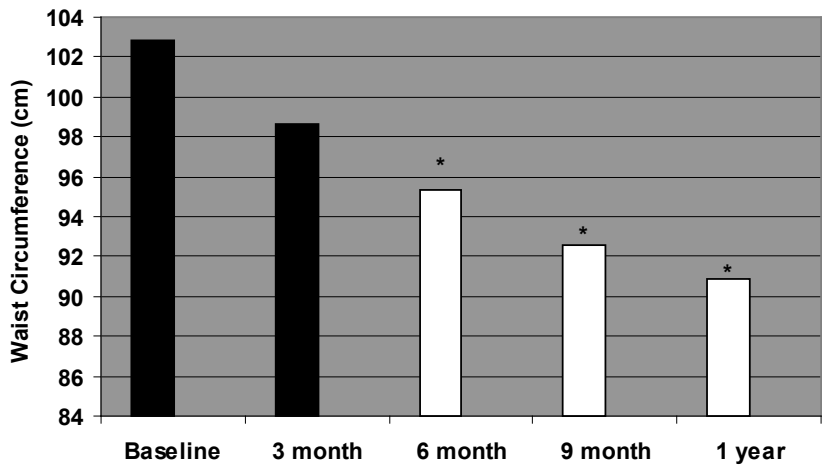

*significant difference versus baseline

Figure 2: Effect of metformin on waist circumference. Waist circumference

$102.8 \pm 14.3 \mathrm{~cm}$ significantly reduced at 6 month $95.3 \pm 12.2 \mathrm{~cm}(p=0.004)$, at

9 month $92.6 \pm 12.7 \mathrm{~cm}(p<0.001)$ and at 1 year $90.9 \pm 12.5 \mathrm{~cm}(p<0.001)$.

\begin{tabular}{|l|c|c|c|}
\hline Body weight reduction & 6 month & 9 month & 1 year \\
\hline Body weight & $r=0.552$ & $r=0.515$ & $r=0.599$ \\
\hline Body Mass Index & $r=0.372^{*}$ & $r=0.501$ & $r=0.449$ \\
\hline Waist circumference & $r=0.569$ & $r=0.549$ & $r=0.573$ \\
\hline Fasting serum insulin & $r=0.723$ & $r=0.523$ & $r=0.588$ \\
\hline 2-h PGL serum insulin & $r=0.471$ & $r=0.539$ & $r=0.512$ \\
\hline HOMA-IR & $r=0.663$ & $r=0.423$ & $r=0.495$ \\
\hline
\end{tabular}

All correlations are statistically significant: $p<0.001,{ }^{*} p<0.01$

Table 2: Pearson or Spearman correlation coefficient $r$ between body weight reduction at 6,9 month and at 1 year and body weight, body mass index, wais circumference, fasting serum insulin, 2-h PGL serum insulin and HOMA-IR at baseline; $P G L=$ post glucose load; $H O M A-I R=$ homeostasis model assessment of insulin resistance.

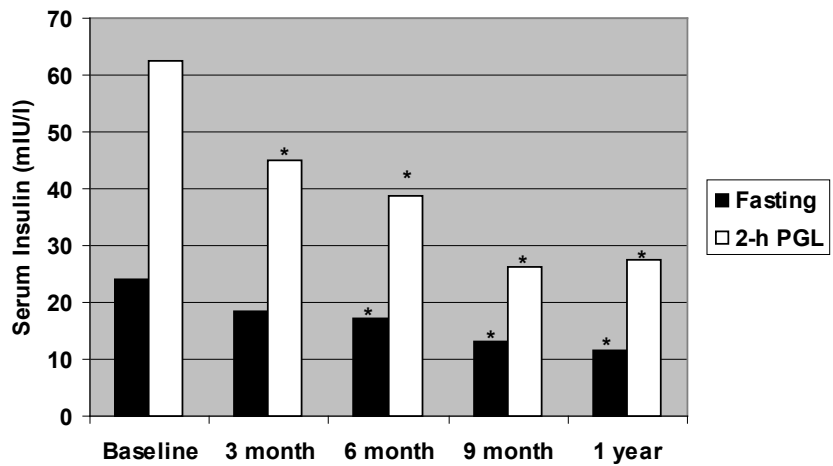

*significant difference versus baseline

Figure 3: Effect of metformin on serum insulin. Fasting serum insulin $24.1 \pm$ $15.3 \mathrm{mIU} / \mathrm{l}$ significantly reduced at 6 month $17.2 \pm 8.8 \mathrm{mlU} / \mathrm{l}(p=0.012)$, at 9 month $13.2 \pm 6.9 \mathrm{mIU} / \mathrm{l}(p<0.001)$ and at 1 year $11.6 \pm 6.0 \mathrm{mIU} / \mathrm{l}(p<0.001) ; 2-\mathrm{h}$ $\mathrm{PGL}$ serum insulin $62.6 \pm 43.2 \mathrm{mlU} / \mathrm{l}$ significantly reduced at 3 month $45.1 \pm$ $26.6 \mathrm{mlU} / \mathrm{l}(\mathrm{p}=0.040)$, at 6 month $38.7 \pm 29.0 \mathrm{mlU} / \mathrm{l}(\mathrm{p}=0.026)$, at 9 month 26.1 $\pm 13.8 \mathrm{mlU} / \mathrm{l}(\mathrm{p}<0.001)$ and at 1 year $27.6 \pm 21.6 \mathrm{mlU} / \mathrm{l} \quad(p<0.001) ; P G L=$ post glucose load.

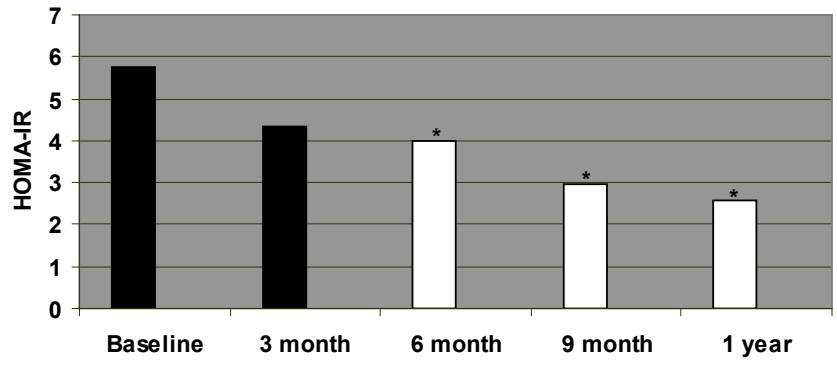

*significant difference versus baseline

Figure 4: Effect of metformin on HOMA-IR. HOMA-IR $5.74 \pm 3.77$ significantly reduced at 6 month $4.01 \pm 2.13(p=0.011)$, at 9 month $2.97 \pm 1.49(p<0.001)$ and at 1 year $2.58 \pm 1.40(p<0.001)$; HOMA-IR: homeostasis model assessment of insulin resistance

weight reduction at 6,9 month and at 1 year with body weight, BMI, waist circumference, fasting serum insulin, 2-h PGL serum insulin and HOMA-IR at baseline is presented in Table 2. Fasting serum insulin was significantly reduced at 6,9 month and at 1 year. At 1 year it was significantly lower in comparison to that of 6 month ( $\mathrm{p}=0.007) .2$-h PGL serum insulin was significantly reduced at 3, 6, 9 month and at 1 year. At 1 year it was significantly lower in comparison to that of 3 month $(\mathrm{p}=0.024)$ (Figure 3 ). HOMA-IR was significantly reduced at 6 , 9 month and at 1 year. At 1 year it was significantly lower in comparison to that of 6 month $(\mathrm{p}=0.006)$ (Figure 4$)$. The fasting plasma glucose 
Citation: Kamenova P, Atanasova I, Kirilov G (2014) Metformin Reduces Cardiometabolic Risk Factors in People at High Risk for Development of Type 2 Diabetes and Cardiovascular Disease. J Diabetes Metab 5: 470 doi:10.4172/2155-6156.1000470

Page 4 of 6

\begin{tabular}{|c|c|c|c|c|c|}
\hline Variables & Baseline & 3 month & 6 month & 9 month & 1 year \\
\hline Fasting PG (mmol/l) & $5.40 \pm 0.53$ & $5.07 \pm 0.52$ & $5.04 \pm 0.40$ & $4.88 \pm 0.52$ & $4.74 \pm 0.50$ \\
\hline$p$ & & $0.029^{*}$ & $0.002^{*}$ & $<0.001^{*}$ & $<0.001^{*}$ \\
\hline Total cholesterol $(\mathrm{mmol} / \mathrm{l})$ & $5.78 \pm 0.76$ & $5.46 \pm 0.94$ & $5.11 \pm 1.04$ & $4.67 \pm 0.95$ & $4.87 \pm 0.86$ \\
\hline$p$ & & 0.265 & $0.009^{*}$ & $<0.001^{*}$ & $<0.001^{*}$ \\
\hline LDL cholesterol (mmol/l) & $3.61 \pm 0.76$ & $3.32 \pm 0.93$ & $3.27 \pm 1.04$ & $2.77 \pm 0.65$ & $2.74 \pm 0.94$ \\
\hline $\mathrm{p}$ & & 0.309 & 0.282 & $0.007^{*}$ & $0.001^{*}$ \\
\hline HDL cholesterol (mmol/l) & $1.10 \pm 0.34$ & $1.28 \pm 0.34$ & $1.23 \pm 0.28$ & $1.20 \pm 0.26$ & $1.44 \pm 0.28$ \\
\hline $\mathrm{p}$ & & 0.114 & 0.214 & 0.403 & $<0.001^{*}$ \\
\hline Triglycerides $(\mathrm{mmol} / \mathrm{l})$ & $2.60 \pm 1.74$ & $2.17 \pm 1.35$ & $1.97 \pm 0.90$ & $1.55 \pm 0.69$ & $1.44 \pm 0.53$ \\
\hline $\mathrm{p}$ & & 0.347 & 0.083 & $0.004^{*}$ & $<0.001^{*}$ \\
\hline Systolic BP (mm Hg) & $131 \pm 18$ & $128 \pm 15$ & $125 \pm 11$ & $121 \pm 15$ & $119 \pm 12$ \\
\hline $\mathrm{p}$ & & 0.426 & 0.057 & $0.008^{*}$ & $<0.001^{*}$ \\
\hline Diastolic BP (mm Hg) & $85 \pm 11$ & $84 \pm 9$ & $81 \pm 8$ & $78 \pm 8$ & $76 \pm 6$ \\
\hline $\mathrm{p}$ & & 0.711 & 0.072 & $<0.001^{*}$ & $<0.001^{*}$ \\
\hline
\end{tabular}

* significant difference versus baseline.

Table 3: Effect of metformin treatment on Fasting PG, Total cholesterol, LDL cholesterol, HDL cholesterol, Triglycerides, Systolic BP, Diastolic BP at 3, 6 , 9 month and at 1 year; $P G=$ plasma glucose; $L D L=$ low density lipoprotein; $H D L=$ high density lipoprotein; $B P=$ blood pressure

was significantly decreased at 3 month, this effect continued to the end of the study and at 1 year it was more pronounced compared to the 3 month ( $\mathrm{p}=0.036$ ). The effect of metformin on fasting plasma glucose, total cholesterol, LDL cholesterol, HDL cholesterol, triglycerides, systolic and diastolic blood pressure is presented in Table 3.

\section{Discussion}

People with hyperinsulinaemia, normal glucose tolerance and MS included in our study represent a high-risk group for development of T2D and CVD. They were young and at middle age, under 60 years of old, socially active people. Their main complaint was the increase in body weight. Some of them had gained a lot of kilograms - between 10 and 30 for a short period of time up to 1 year, others had noticed a gradually increase in body weight up to 50 kilograms for a longer period. A majority of participants had experienced episodes of tremor, increased sweating and tachycardia on fasting or after ingestion of sugar or carbohydrate food. Tachycardia at rest, peripheral oedema or unmotivated nervousness were the other concerns. Some of women had an irregular menstrual cycle and/or impossibility to become pregnant.

A large scale prospective multicenter study for prevention of diabetes, Diabetes Prevention Program (DPP), underlines the significance of lifestyle intervention, including diet and physical activity for reduction in body weight and incidence of diabetes in people with obesity and impaired glucose tolerance $[3,4]$. Intensive diet and physical activity independently of each other reduces the risk of diabetes. The benefit of lifestyle intervention is greater in people above 60 years and those with family history of diabetes, hypertension and obesity [2]. Before enrollment in our study all individuals were unsatisfied by lack of weight loss with conventional medical care-hypocaloric diet and individual physical activity in prior year and they were not motivated to keep on the lifestyle intervention.

Metformin has been shown to decrease body weight and incidence of T2D with greatest impact in people under 60 years of old like those participated in our study $[3,26]$. We found that at least 6 months were needed so as metformin significantly reduced body weight, BMI and waist circumference. The reduction in body weight, BMI and waist circumference continued to the end of the observation and it was no more pronounced compared to the 6 month. The significant decrease in BMI is observed at 6 month of metformin treatment added to an individual diet and physical activity in obese hyperinsulinaemic adolescents between 9 and 17 years old [16]. There is no a significant difference in the volume of visceral fat and indices of fat distribution measured by computed tomography scan between placebo and metformin, applied for a shorter period of 3 months [18]. Metformin treatment for a longer period of 36.1 months without controlled diet results in a reduction of BMI especially in women with polycystic ovary syndrome and MS at the beginning of the treatment [11]. Even after 10 years of metformin treatment the reduction in body weight has been kept and the incidence of diabetes has been reduced by $18 \%$ in comparison to placebo [4].

Following metformin treatment, the mean body weight reduction at 6 month of $7.7 \mathrm{~kg}$ and at 1 year of $12.1 \mathrm{~kg}$ in our study was greater than described in DPP by both-lifestyle intervention and metformin. The average weight loss at 6 month is 2.1 and $5.6 \mathrm{~kg}$ in metformin and lifestyle intervention groop that makes the lifestyle intervention superior compared to metformin. The intensive diet and 150 minutes physical exercise per week leads to a mean body weight reduction of $6.8 \mathrm{~kg}$ in the first year [3]. Metformin in combination with carbohydrate-modified hypocaloric diet leads to a body weight loss at 6 month of $7.5 \mathrm{~kg}$ and at 1 year of $10.7 \mathrm{~kg}$ in nondiabetic women with hyperinsulinaemia [5].

We observed, that body weight reduction was as more pronounced, as the higher were the baseline values of body weight, BMI, waist circumference, fasting insulin, 2-h PGL insulin and HOMA-IR. At 1 year, the maximal weight loss of $22 \mathrm{~kg}$ was in a man with a baseline body weight of $121 \mathrm{~kg}$, BMI of $31.5 \mathrm{~kg} / \mathrm{m}^{2}$, fasting insulin of $56.3 \mathrm{mIU} / \mathrm{l}$ and HOMA-IR of 15.01 and the minimal weight loss of $4 \mathrm{~kg}$ was in a woman with a baseline body weight of $75 \mathrm{~kg}$, BMI of $27 \mathrm{~kg} / \mathrm{m}^{2}$, fasting insulin of $9.4 \mathrm{mIU} / \mathrm{l}$ and HOMA-IR of 1.83 . These findings confirmed the previously published data about the most pronounced effect of metformin therapy on body weight in individuals with higher values of BMI [3]. We could add that the reduction in body weight was as expressed as the greater were fasting and $2 \mathrm{~h}$ PGL hyperinsulinaemia and insulin resistance.

Our results indicated that effect of metformin on hyperinsulinaemia and insulin resistance was increasing over the time of observation. The decrease in fasting serum insulin and HOMA-IR was significant at 
6,9 month and at 1 year it was the most pronounced. The significant effect of metformin on 2-h PGL serum insulin was noticed at 3, 6, 9 month and at 1 year it was the most expressed. In patients with heart failure and insulin resistance, defined by fasting insulin resistance index (FIRI), metformin reduces insulin resistance in comparison to placebo for a shorter period of 4 months [23]. In 19 individuals with MS and T2D, metformin treatment at a dose of $1.7 \mathrm{~g}$ daily for a period of 6 weeks does not have a significant effect on HOMA-IR and fasting plasma glucose [27]. In concordance to our data, as reduction in BMI, metformin reduces fasting, 2-h PGL serum insulin and HOMA-IR, having in mind that it is applied in combination with individual diet and physical activity [16]. In contrast to our findings, in obese impaired glucose tolerant and normal glucose tolerant people with risk factors for T2D from DPP study, there is no a significant effect of metformin on fasting and 2-hour insulin secretion for a period of 1 year [10]. There is a study in individuals with MS showing the effect of metformin on insulin resistance at a dose of $1 \mathrm{~g}$ daily for a period of 3 months [19].

Our study confirmed the antihyperglycaemic, but not hypoglycaemic action of metformin. The effect of metformin on fasting plasma glucose was increasing over the time of observation. Metformin significantly reduced fasting plasma glucose within the normoglycaemic range at 3 month, this effect continued to the end of the observation and it was the most pronounced at 1 year without reaching hypoglycaemic values. Effect of metformin on fasting plasma glucose at 3 month in first degree relatives of type 2 diabetics with normal glucose tolerance, MS and obesity is noticed [17,21]. Metformin alone or added to diet reduces fasting plasma glucose in people with overweight, obesity, MS, normal glucose tolerance with risk factors for diabetes and prediabetes over a 1 year period $[8,10]$.

The effect of metformin on total cholesterol was significant at 6,9 month and at 1 year. The decrease in LDL cholesterol and triglycerides was significant at 9 month and at 1 year. HDL cholesterol was significantly increased at 1 year. The decrease in systolic and diastolic blood pressure was significant at 9 month and at 1 year. A 1 -year metformin treatment reduces systolic blood pressure, total and LDL cholesterol with no effect on body weight, HDL cholesterol and triglycerides in people with normal and impaired glucose tolerance and impaired fasting blood glucose in a BIGPRO1 Trial [10]. Metformin decreases total cholesterol, LDL cholesterol, Cholesterol: HDL cholesterol ratio and systolic blood pressure and increases HDL cholesterol in double blind randomized controlled studies in women with polycystic ovary syndrome and first line relatives of type 2 diabetics with normal glucose tolerance and MS for a shorter period of 3 months $[17,18,21]$.

A 10-year analysis of effectiveness of three therapeutic regimens (metformin, lifestyle change and placebo) in DPP shows a significant reduction in systolic blood pressure, diastolic blood pressure, LDL cholesterol and triglycerides and a significant increase in HDL cholesterol in all treatment groups similarly and it is noticed less medications for dyslipidaemia and hypertension in the group with intensive lifestyle change [9]. At the beginning of our study 22 individuals took antihypertensive drugs. At 1 year of metformin treatment 12 of them reduced the number of antihypertensive drugs and 8 individuals stopped medications. At the end of the study all 5 individuals who were taking drugs for dyslipidaemia discontinued their use. There was no CVD events occurred during the study.

Generally, the metformin treatment was well tolerated. We did not observe serious side effects, hypoglycaemias and changes in routine biochemical parameters. Some people complained of mild gastrointestinal symptoms like diarrhoea, flatulation or nausea at the beginning of the treatment, that dissapeared during titration period and did not cause discontinuation of the treatment in any of them.

According to the Position Statement of the American Diabetes Association and the European Association for the Study of Diabetes, metformin is a first-line therapy for T2D [28]. Having in mind the role of metformin for diabetes prevention, the American Association of Clinical Endocrinologists has recommended metformin for treatment of high-risk individuals with impaired glucose tolerance and impaired fasting blood glucose [2]. Reduction of factors leading to the cardiovascular complications like systolic blood pressure, diastolic blood pressure and triglycerides and improvement of HDL cholesterol and endothelial function leads to the conclusion that metformin could be applied for the treatment of MS $[11,19,20]$.

\section{Conclusion}

People who have hyperinsulinaemia, normal glucose tolerance and MS are at high risk for development of T2D and CVD, that makes the treatment of cardiometabolic risk factors mandatory. Usually we treat obesity with lifestyle intervention, hypertriglyceridaemia and low HDL cholesterol with fibrates, high LDL cholesterol with statins and hypertension with antihypertensive drugs. Our open-label one year prospective observational clinical study shows that treatment with metformin alone, without intensive diet and physical activity, in people with hyperinsulinaemia, normal glucose tolerance and MS could reduce cardiometabolic risk factors as visceral obesity, hyperinsulinaemia, insulin resistance, dyslipidaemia and arterial hypertension and supports the hypothesis that metformin could be applied for prevention of T2D and CVD. As this study have some limitations referred to a small sample size and lack of a control group, larger studies should be carried out to investigate the effects of metformin on high-risk individuals for development of T2D and CVD.

\section{References}

1. Wilson PW, Meigs JB (2008) Cardiometabolic risk: a Framingham perspective Int J Obes (Lond) 2: S17-20.

2. Lukacova-Zib I, Gopalakrishnan G (2010) Therapeutic options for the prevention of type 2 diabetes mellitus in the metabolic syndrome. Mt Sinai $J$ Med 77: 524-532.

3. Knowler WC, Barrett-Conner E, Fowler SE, Hamman RF, Lachin JM, et al (2002) Reduction in the incidence of type 2 diabetes with lifestyle intervention or metformin. N Engl J Med 346: 393-403.

4. Diabetes Prevention Program Research Group, Knowler WC, Fowler SE Hamman RF, Christophi CA, et al. (2009) 10-year follow-up of diabetes incidence and weight loss in the Diabetes Prevention Program Outcomes Study. Lancet 374: 1677-1686.

5. Mogul HR, Peterson SJ, Weinstein BI, Zhang S, Southren AL (2001) Metformin and carbohydrate-modified diet: A novel obesity treatment protocol: Preliminary findings from a case series of nondiabetic women with midlife weight gain and hyperinsulinemia. Heart Dis 3: 285-292.

6. Harden KA, Cowan PA, Velasquez-Mieyer P, Patton SB (2007) Effects of lifestyle intervention and metformin on weight management and markers of metabolic syndrome in obese adolescents. J Am Acad Nurse Pract 19: 368377.

7. Boyle JG, MoKay GA, Fisher M (2010) Drugs for diabetes: part 1 metformin. $\mathrm{Br}$ J Cardiol 17: 231-234.

8. Andreadis EA, Katsanou PM, Georgiopoulos DX, Tsourous GI, Yfanti GK, et al. (2009) The effect of metformin on the incidence of type 2 diabetes mellitus and cardiovascular disease risk factors in overweight and obese subjects-the Carmos study. Exp Clin Endocrinol Diabetes 117: 175-180.

9. Diabetes Prevention Program Outcomes Study Research Group, Orchard TJ, Temprosa M, Barrett-Connor E, Foyler SE, et al. (2013) Long-term effects of the Diabetes Prevention Program interventions on cardiovascular risk factors: a report from the DPP Outcomes Study. Diabet Med 30: 46-55. 
Citation: Kamenova P, Atanasova I, Kirilov G (2014) Metformin Reduces Cardiometabolic Risk Factors in People at High Risk for Development of Type 2 Diabetes and Cardiovascular Disease. J Diabetes Metab 5: 470 doi:10.4172/2155-6156.1000470

10. Fontbonne A, Diouf I, Baccara-Dinet M, Eschwege E, Charles MA (2009) Effects of 1 -year treatment with metformin on metabolic and cardiovascular risk factors in non-diabetic upper- body obese subjects with mild glucose anomalies: aposthocanalysis of the BIGPRO1 trial. Diabetes Metab 35: 385-391.

11. Cheang KI, Huszar JM, Best AM, Sharma S, Essah PA, et al. (2009) Long-term effect of metformin on metabolic parameters in the polycystic ovary syndrome. Diab Vasc Dis Res 6: 110-119.

12. Krstevska B, Dimitrovski Ch, Pemovska G, Misevska S, Dimova Z, et al. (2006) Metformin improves menstrual patterns, endocrine and metabolic profile in obese hyperinsulinemic women with a polycystic ovary syndrome. Prilozi 27 : 57-66.

13. Oppelt PG, Mueller A, Jentsch K, Kronawitter D, Reissmann C, et al. (2010) The effect of metformin treatment for 2 years without caloric restriction on endocrine and metabolic parameters in women with polycystic ovary syndrome. Exp Clin Endocrinol Diabetes 118: 633-637.

14. Fux Otta C, Wior M, Iraci GS, Kaplan R, Torres D, et al. (2010) Clinical, metabolic, and endocrine parameters in response to metformin and lifestyle intervention in women with polycystic ovary syndrome: a randomized, doubleblind, and placebo control trial. Gynecol Endocrinol 26: 173-178.

15. Saxena P, Prakash A, Nigam A (2010) Effect of metformin therapy on 2-h postglucose insulin levels in patients of polycystic ovarian syndrome. J Hum Reprod Sci 3: 139-142.

16. Atabek ME, Pirgon O (2008) Use of metformin in obese adolescents with hyperinsulinemia: a 6-month, randomized, double-blind, placebo-controlled clinical trial. J Pediatr Endocrinol Metab 21: 339-348.

17. LimaLM, Wiemsperger N, Kraemer-Aguiar LG, Bouskela E (2009) Short-term treatment with metformin improves the cardiovascular risk profile in firstdegree relatives of subjects with type 2 diabetes mellitus who have a metabolic syndrome and normalglucose tolerance without changes in C-reactive protein or fibrinogen. Clinics (Sao Paolo) 64: 415-420.

18. Lord J, Thomas R, Fox B, Acharya U, Wilkin T (2006) The effect of metformin on fat distribution and the metabolic syndrome in women with polycystic ovary syndrome--a randomised, double-blind, placebo-controlled trial. BJOG 113: 817-824.
19. Vitale C, Mercuro G, Cornoldi A, Fini M, Volterrani M, et al. (2005) Metformin improves endothelial function in patients with metabolic syndrome. J Intern Med 258: 250-256.

20. Meaney E, Vela A, Samaniego V, Meaney A, Asbún J, et al. (2008) Metformin arterial function, intima-media thickness and nitroxidation in metabolic syndrome: the mefisto study. Clin Exp Pharmacol Physiol 35: 895-903.

21. de Aguiar LG, Bahia LR, Vilela N, Laflor C, Sicuro F, et al. (2006) Metformin improves endothelial vascular reactivity in first-degree relatives of type 2 diabetic patients with metabolic syndrome and normal glucose tolerance. Diabetes Care 29: 1083-1089.

22. Fitch K, Abbara S, Lee H, Stavrou E, Sacks R, et al. (2012) Effects of lifestyle modification and metformin on atherosclerotic indices among HIV-infected patients with the metabolic syndrome. AIDS 26: 587-597.

23. Wong AK, Symon R, AIZadjali MA, Ang DS, Ogston S, et al. (2012) The effect of metformin on insulin resistance and exercise parameters in patients with heart failure. Eur J Heart Fail 14: 1303-1310.

24. Alberti KG, Zimmet P, Shaw J; IDF Epidemiology Task Force Consensus Group (2005) The metabolic syndrome--a new worldwide definition. Lancet 366: 10591062.

25. Haffner SM, Kennedy E, Gonzalez C, Stern MP, Miettinen H (1996) A prospective analysis of the HOMA model. The Mexico City Diabetes Study. Diabetes Care 19: 1138-1141.

26. Herman WH, Hoerger TJ, Brandle M, Hicks K, Sorensen S, et al. (2005) The cost-effectiveness of lifestyle modification or metformin in preventing type 2 diabetes in adults with impaired glucose tolerance. Ann Intern Med 142: 323 332.

27. Castro Cabezas M, van Wijk JP, Elte JW, Klop B (2012) Effects of metformin on the regulation of free Fatty acids in insulin resistance: a double-blind, placebocontrolled study. J Nutr Metab 2012: 394623.

28. Inzucchi SE, Bergenstal RM, Buse JB, Diamant M, Ferrannini E, et al. (2012) Management of hyperglycaemia in type 2 diabetes. A patient-centered Approach Position Statement of the American Diabetes Association (ADA) and the European Association for the Study of Diabetes (EASD). Diabetes Care 35: 1364-1379. 